\title{
Impact of slip boundary on sloshing motions in partially filled containers
}

\author{
AHMAD Salman* \& YUE BaoZeng \\ Department of Mechanics, School of Aerospace Engineering, Beijing Institute of Technology, Beijing 100081, China
}

Received April 16, 2011; accepted May 31, 2011

\begin{abstract}
Sloshing is a kind of fluid motion inside partially filled containers. In spacecraft and other partially filled moving containers, sloshing plays an important role. The contact line between the fluid and solid boundary affects the fluid movement and sloshing during motion. A physical model for steady fluid flow with a partial slip boundary is presented and equations for this model are derived for cylindrical (tube-shaped) tanks. This gives a nonlinear system of differential equations that is solved numerically by using a Successive Over-Relaxation (SOR) technique and graphical results are shown. Variations in steady fluid flow are observed with changes in the slip length and some useful results are derived. The effects on the microscopic radius of the fluid layer in a capillary tube are also shown through graphical results.
\end{abstract}

sloshing of fluids, partial slip boundary, disjoining pressure, contact line, finite difference method, successive over-relaxation technique

Citation: Ahmad S, Yue B Z. Impact of slip boundary on sloshing motions in partially filled containers. Chinese Sci Bull, 2011, 56: 2674-2678, doi: $10.1007 / \mathrm{s} 11434-011-4644-3$

Free surface sloshing in moving containers has many engineering applications, such as tanks in trucks on roads, fluid oscillation in storage tanks caused by earth-quakes [1,2], sloshing of liquid-cargo vessels in ships and the movement of liquid in spacecrafts. Sloshing of fluids in moving containers is comparatively more complex. The frequency of disturbance and shape of the container play an important role. A number of complex motions, including non-planar, rotational, irregular beating, quasi periodic and chaotic motion, can be observed at the free surface during sloshing. An in-depth knowledge about the factors that reduce sloshing can maximize the control of fluid-carrying tankers and hence may improve spacecraft technology.

The stability of vehicles [3] has been the main interest of researchers in tackling the difficulties caused by sloshing during motion. Rebouillat et al. [4] provided a nice review of results and applications of sloshing models in aerospace engineering, naval technology and other industries. Zhu et

*Corresponding author (email: salman_bzm@yahoo.com) al. [5] used the level set method for rectangular tanks to find the pressure caused by sloshing. A detailed study of previous theoretical and experimental research on sloshing has been presented by Ibrahim [6]. Yue [7] discussed sloshing in rectangular and circular containers and derived numerical results for pitching excitation. Yue et al. [8] also derived some important results using an arbitrary Lagrange-Euler description. Gavrilyuk [9] presented useful results about sloshing in vertical circular cylinders for nonlinear resonant waves. Cheng et al. [10] and He et al. [11] discussed sloshing in low-gravity situation. Cheng et al. [10] found very useful results for sloshing parameters by observing the changes in frequency and transverse force with sloshing parameter variation. In the ideal case, the solid boundaries of a container experience no slipping, although most of the time some slippage on the boundaries will occur. Sloshing of fluids with such boundaries makes the system more complex. In more recent times, slippage has been discussed in the case of non-Newtonian fluids with solid/gas interfaces and for some special surfaces [12-15]. Evidence for slip- 
page in Newtonian fluids has been studied for several years, but its use in experiments was first discussed in 2000 [16]. In these studies, many useful results have been discovered [17-20]. It has been noticed that slippage depends upon the shear stress [21]. Zhou et al. [22] proposed the velocity-slip criteria to study slip impact on the boundary walls. Santra et al. [23] discussed axisymmetric stagnation point flow over a slip boundary layer, where this layer is produced using a non-Newtonian liquid film with variable thickness. Finding the accurate slip-length is not an easy task. Li et al. [24] proposed a method for finding the slip-length by using a rheometer. The contribution of the present work is the combination of sloshing with partial slip boundary conditions. We study the steadiness of fluid thickness in the presence of slippage. For this purpose, we introduced a slip parameter with variable length. David et al. [25] derived the fluid thickness of steady fluid flow for sloshing motion by ignoring slippage at the boundary. We present a model with partial slip boundary conditions and derive our results numerically for steady fluid flow. We observe that, by ignoring the slip parameter, the numerical results derived in our work match the results founded by David et al. [25]. In the second section, mathematical formulations are presented for the model considered with partial slip boundary conditions, providing a nonlinear system of differential equations. Analytical solutions for such systems of highly nonlinear differential equations is difficult to achieve, so the finite difference method (FDM) with the SOR method is used to find the numerical results in third section. Graphical results and a table help us to show the effect of the slip boundary condition on steady fluid flow and give useful information about slippage. The last section contains our conclusions, and some interesting ideas for future work are proposed.

\section{Mathematical formulations}

Consider a fluid meniscus meeting the boundary of a container at a given contact angle $\theta$. The model of flow is depicted in Figure 1 and is expressed by the following equations.

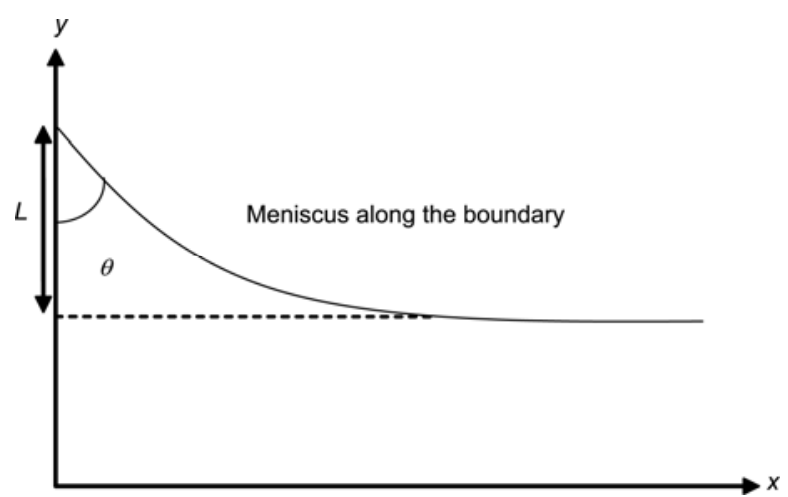

Figure 1 Model of fluid under consideration.
Within fluid the hydrostatic pressure is given as

$$
p(y)=-\rho g y=-\sigma \kappa,
$$

where $g$ is gravitational acceleration, $\rho$ is the density of the fluid, $\sigma$ is surface tension, $p$ is pressure and $\kappa$ is curvature. For $\kappa$, we have

$$
\kappa=-\frac{\mathrm{d} \theta}{\mathrm{d} s}=-\frac{\mathrm{d} \theta}{\mathrm{d} y} \frac{\mathrm{d} y}{\mathrm{~d} s},
$$

where $s$ is the arc length. Since $\frac{\mathrm{d} y}{\mathrm{~d} s}=\cos \theta$, we get

$$
\rho g y=-\sigma \cos \theta \frac{\mathrm{d} \theta}{\mathrm{d} y} .
$$

Integrating with respect to $y$ gives

$$
\frac{\rho g y^{2}}{2 \sigma}=1-\sin \theta \text {. }
$$

Since $\theta=\theta_{e}$ at $y=L$, we have

$$
\frac{\rho g L^{2}}{2 \sigma}=1-\sin \theta_{e},
$$

where $\theta_{e}$ is the equilibrium or static contact angle. The radius $R$ is then given by

$$
R=\frac{\sigma}{\rho g L}=\frac{l_{c}}{\sqrt{2\left(1-\sin \theta_{e}\right)}},
$$

where the capillary length is

$$
l_{c}=\sqrt{\frac{\sigma}{\rho g}} .
$$

Using the Lennard-Jones potential for molecules interacting in an ideal gas, we introduce disjoining pressure $\Pi$ to simulate the macroscopic contact angle between the fluid and substrate. Thus the total pressure is given by

$$
p=-\sigma \kappa-\Pi \cong-\sigma h_{x x}-\Pi \text {. }
$$

The disjoining pressure is given by

$$
\Pi=B\left[\left(\frac{h_{*}}{h}\right)^{n}-\left(\frac{h_{*}}{h}\right)^{m}\right],
$$

where $h_{*}$ is the wetting layer thickness and $h$ is the height of the steady fluid thickness.

The exponential parameters $n>m>1$ are arbitrary, and in our case we use $(n, m)=(4,3)$. Moreover, the disjoining coefficient $B$ is given by

$$
B=\frac{(n-1)(m-1)}{h_{*}(n-m)} \sigma\left(1-\cos \theta_{e}\right) \cong \frac{(n-1)(m-1)}{2 h_{*}(n-m)} \sigma \theta_{e}^{2} .
$$

Introducing the lubrication approximation [26], we have 


$$
h^{\prime}(x)<<1
$$

and $p(x)=-\sigma \kappa-\sigma / R-\Pi \cong-\sigma h_{x x}-\sigma / R-\Pi$.

As we have already discussed, this is an ideal situation in which there is no slippage between the boundary or substrate of the fluid container and the fluid itself. Most substrates, however, do experience some slippage that until now we have neglected. We next introduce the slip boundary condition for a fuel container experiencing sloshing motion. This will provide us with real and useful results for the stability of containers on spacecraft and moving vehicles. An explanation of the model with a partial slip boundary condition is given below.

In Figure 2, for the no-slip boundary conditions in (a) the relative velocity, $\vec{V}$, between the plate and the fluid is zero at the plate. When slip occurs (b) at the plate, $\vec{V}=\vec{V}_{s}>0$. Here, slip is characterized by the slip length $\lambda$. It should be noted that the slip length is related to the wall velocity $\vec{V}_{s}$ through the equation $\vec{V}_{s}=\lambda \frac{\partial \vec{V}}{\partial y}$, where $\frac{\partial \vec{V}}{\partial y}$ is the local shear rate. If the plate is moving with some velocity $\vec{U}_{1}$ then the difference between the velocity of the fluid flow and the velocity of the plate gives the relative velocity at the plate and is related to the slip length by $\vec{V}-\vec{U}_{1}=\lambda \frac{\partial \vec{V}}{\partial y}$.

For small Reynolds number, the Navier-Stokes equations give

$$
u_{y y}=\frac{p_{x}}{\mu}
$$

where $\mu$ is viscosity, with boundary conditions:

$$
\begin{gathered}
u(x, 0)=\lambda \frac{\partial u}{\partial y} \quad \text { (partial-slip) } \\
u_{y}(x, h)=0 \text { (no-stress). }
\end{gathered}
$$

(a)

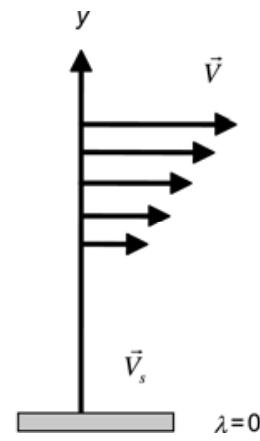

(b)

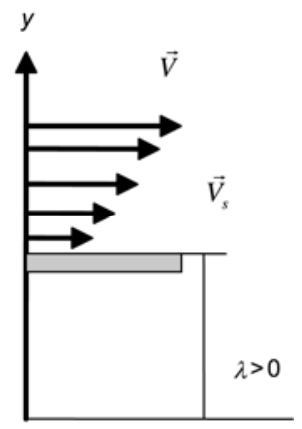

Figure 2 Model for the shear dependence of partial slip boundary. (a) Flow of fluid with no-slip boundary; (b) flow of fluid with partial-slip boundary.
Integrating twice with respect to $y$ and applying the boundary conditions we get

$$
u(x, y)=\frac{p_{x}}{\mu}\left[\frac{1}{2} y^{2}-h y-\lambda h\right] .
$$

The flux is given by

$$
Q=\int_{0}^{h(x)} u(x, y) \mathrm{d} y=-\frac{p_{x} h^{2}}{\mu}\left(\frac{h}{3}+\lambda\right) .
$$

Continuity requires that

$$
Q=U\left(h-h_{\infty}\right),
$$

where $h_{\infty}$ is coating thickness and $U$ is speed. Thus

$$
-p_{x}=\mu U \frac{h-h_{\infty}}{h^{2}\left(\frac{h}{3}+\lambda\right)} .
$$

Using this value for $p_{x}$ gives

$h_{x x x}+\frac{3 \theta_{e}^{2}}{h_{*}^{2}}\left[3\left(\frac{h_{*}}{h}\right)^{4}-4\left(\frac{h_{*}}{h}\right)^{5}\right] h_{x}=\frac{3 \mu U\left(h-h_{\infty}\right)}{\sigma h^{2}(h+3 \lambda)}$,

$h^{3} h_{x x x}+3 \theta_{e}^{2} h_{*}^{2}\left[\frac{3}{h}-\frac{4 h_{*}}{h^{2}}\right] h_{x}=\frac{3 \mu U h\left(h-h_{\infty}\right)}{\sigma(h+3 \lambda)}$.

We now introduce the non-dimensional variables $h=$ $h_{1} \bar{h}, \quad x=L \bar{x}$, Capillary number $=C a=\frac{\mu U}{\sigma}$ and $L=\frac{h_{1}}{(3 C a)^{1 / 3}}$. Thus eq. (1.21) gives

$$
\bar{h}^{3} \bar{h}_{\overline{x x x}}+K\left[\frac{3}{\bar{h}}-\frac{4 \bar{h}_{*}}{\bar{h}^{2}}\right] \bar{h}_{\bar{x}}=\frac{\bar{h}\left(\bar{h}-\bar{h}_{\infty}\right)}{(\bar{h}+3 \bar{\lambda})},
$$

where $\quad K=\frac{3 \theta_{e}^{2} \bar{h}_{*}^{2}}{(3 C a)^{2 / 3}}$.

Finally, removing the bar and simplifying gives

$$
\begin{aligned}
& h^{3} h_{x x x}+3 \lambda h^{2} h_{x x x}+K\left[\frac{3}{h}-\frac{4 h_{*}}{h^{2}}\right] h_{x}+3 \lambda K\left[\frac{3}{h^{2}}-\frac{4 h_{*}}{h^{3}}\right] h_{x} \\
= & h-h_{\infty} .
\end{aligned}
$$

\section{Numerical results}

Eq. (1.23) gives us a highly nonlinear differential system, so we use the FDM to calculate the derivatives below:

$$
\begin{gathered}
\left.h_{x}\right|_{i} \cong \frac{h_{i+1}-h_{i-1}}{2 \Delta x}, \\
\left.h_{x x}\right|_{i} \cong \frac{h_{i-1}-2 h_{i}+h_{i+1}}{(\Delta x)^{2}},
\end{gathered}
$$




$$
\begin{aligned}
\left.h_{x x x}\right|_{i+1 / 2} & \cong \frac{\left.h_{x x}\right|_{i+1}-\left.h_{x x}\right|_{i}}{\Delta x} \\
& =\frac{-h_{i-1}+3 h_{i}-3 h_{i+1}+h_{i+2}}{(\Delta x)^{3}} .
\end{aligned}
$$

For the nonlinear terms $h^{3} h_{x x x}$ and $h^{2} h_{x x x}$, we evaluate $h^{3}$ and $h^{2}$ as pre-factors and define

and

$$
\begin{gathered}
S_{i+1 / 2} \equiv h_{i+1 / 2}^{3} \cong \frac{2 h^{2}{ }_{i} h_{i+1}^{2}}{\left(h_{i}+h_{i+1}\right)}, \\
T_{i+1 / 2} \equiv h^{2}{ }_{i+1 / 2} \cong \frac{2 h^{2}{ }_{i} h_{i+1}}{\left(h^{2}{ }_{i}+h^{2}{ }_{i+1}\right)} .
\end{gathered}
$$

Also, for the disjoining terms we define

$$
f_{i}=\frac{3}{h_{i}}-\frac{4 h_{*}}{h^{2}{ }_{i}}, \text { and } g_{i}=\frac{3}{h_{i}^{2}}-\frac{4 h_{*}}{h_{i}^{3}} \text {. }
$$

Hence eq. (1.23) written in finite difference form becomes

$$
\begin{aligned}
& \left.S_{i+1 / 2} h_{x x x}\right|_{i+1 / 2}+3 \lambda\left(\left.T_{i+1 / 2} h_{x x x}\right|_{i+1 / 2}\right)+K\left(\left.f_{i} h_{x}\right|_{i}\right) \\
& +3 \lambda K\left(\left.g_{i} h_{x}\right|_{i}\right)=h_{i}-h_{\infty} .
\end{aligned}
$$

From the boundary conditions, matching the curvature of the macroscopic radius at the left hand boundary gives $h_{x x}(0)=\frac{1}{R\left(\theta_{e}\right)}$, with $h(0)=h_{0}$. The fixed slope at the right hand boundary is $h^{\prime}(x)=0$. Applying the boundary conditions to the system of equations and using FDM with MATLAB, we get graphical data that expresses the variation in the thickness of steady fluid flow with changes in the partial slip boundary along the substrate.

From Figure 3, we observe that the steady fluid thickness decreases along the horizontal axis and loses the property of being steady. Table 1 is constructed with an error tolerance of $10^{-5}$ and for $n=2000$, where $\theta=0^{\circ}, h_{*}=10^{-6}$ and $R=15$. We observe from the table that the values of $h$ are getting closer together as the values of slip length $\lambda$ increase, which means that at a certain stage there will be negligible or constant difference between two consecutive values of the steady fluid thickness.

Figure 4 presents a graph of fluid thickness for different values of the radius of curvature. The horizontal axis is the image of logarithmic scale to reduce the number of points in the restricted domain. These curves can actually be considered as the input for the radius of curvature, and we can therefore find the variation in radius from the change in fluid thickness.

\section{Discussion and conclusions}

The choice of slip parameter for simple liquids has been

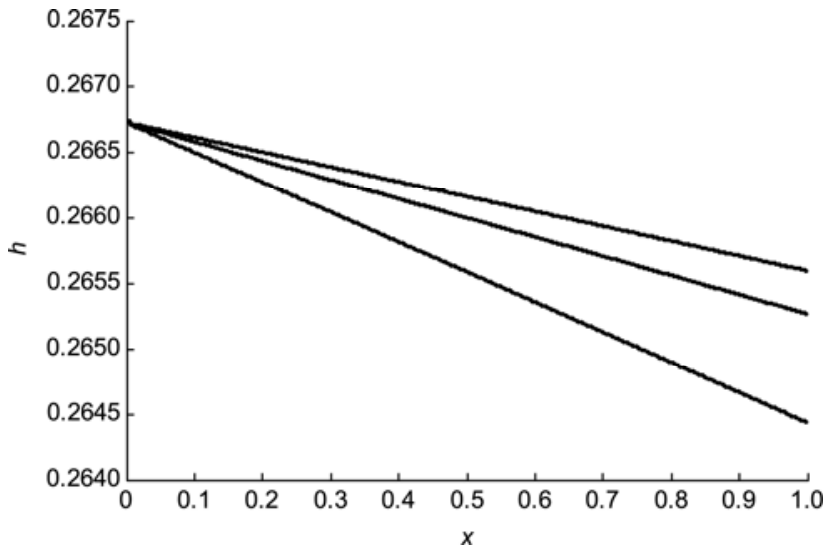

Figrue 3 Upper curve is for $\lambda=0.09$, the middle curve is for $\lambda=0.05$ and the bottom curve is for $\lambda=0$, where $\theta=0^{\circ}$.

Table 1 Steady fluid height variation with slip-length

\begin{tabular}{ll}
\hline$\lambda$ & $h$ \\
\hline 0.0 & 0.2501 \\
0.05 & 0.2566 \\
0.09 & 0.2589 \\
0.1 & 0.2594 \\
0.12 & 0.2601 \\
0.13 & 0.2604 \\
0.14 & 0.2607 \\
0.15 & 0.2610 \\
\hline
\end{tabular}

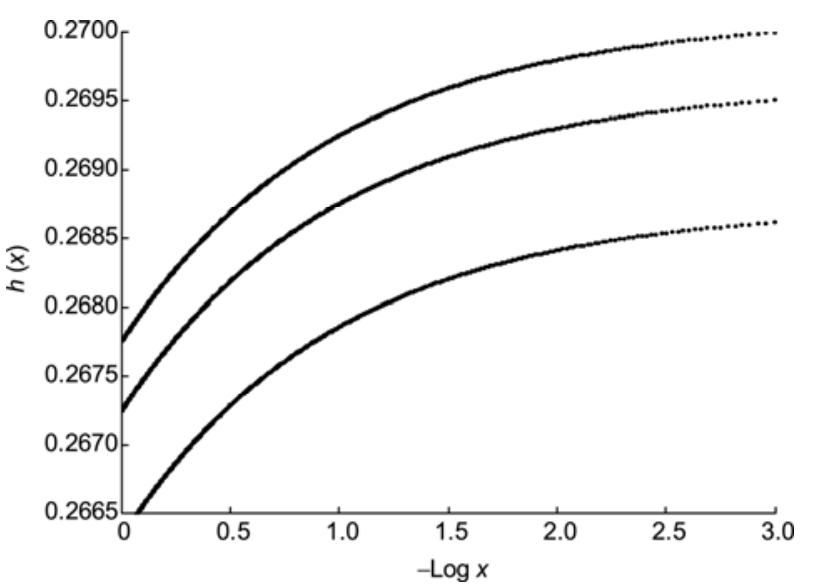

Figure 4 The upper curve is for $R=0.30$, the middle curve is for $R=0.35$ and the bottom curve is for $R=0.5$, where $\theta=\pi / 3$ and $\lambda=0.05$.

found from experiments to determine the results for fluid height [1]. Moreover, the curves in Figure 3 are consistent with the pattern of curves and values as founded by David et al. [25] for no-slip boundary conditions (that is, setting $\lambda=0$ ). This comparison of curves and values verifies our computational work and on the basis of this we have proceeded to get useful results. From Table 1 and Figure 3, we 
conclude that in the absence of external stress, partial slippage helps the flow to remain either steady or unsteady. This means that if fluid inside a fixed container on a vehicle or in a spacecraft is disturbed, then it will continue moving with the same disturbance for a long period if the boundaries exhibit partial slip conditions. More slippage will result in a longer time needed for the fluid to be stable, and thus a partial slip boundary can lead to more complex sloshing motion than a rough solid surface. Care is needed when tackling such problems.

In Table 1, we see that with an increase in slip length $\lambda$, the values of the steady fluid height $h(x)$ also increase. This verifies our results physically, because slippage encourages the fluid to retain its state of flow. Moreover, as we move down Table 1, we observe that the difference between the values of $h(x)$ are getting closer and that at a certain level the steady fluid thickness will either become constant or the difference will become constant. This result is very useful and interesting for the stability of moving fluid containers and has been discussed for the first time in this article.

We have derived these results for a fixed, stress-free boundary. This work can be extended to the case of a boundary that is experiencing motion with some constant or variable acceleration or is under some external stress. Unsteady flows for such boundaries have not yet been discussed. Results for unsteady flows may help us to calculate velocity profiles, frequency, energy dissipation during sloshing, and other parameters that would be useful for adaptive control of spacecraft and partially filled fluid containers.

This work was supported by the Higher Education Commission of Pakistan, National Natural Science Foundation of China (11072030), Ph.D. Programs Foundation of Ministry of Education of China (20080070011), Scientific Research Foundation of Ministry of Education of China for Returned Scholars (20080732040) and Program of Beijing Municipal Key Discipline Construction.

1 Pit R, Hervet H, Léger L. Direct experimental evidence of slip in hexadecane: Solid interfaces. Phys Rev Lett, 2000, 85: 980-983

2 Wu G X. The sloshing of stratified liquid in a two-dimensional rectangular tank. Sci China-Phys Mech Astron, 2011, 54: 2-9

3 Toumi M, Bouazara M, Richard M J. Impact of liquid sloshing on the behavior of vehicles carrying liquid cargo. Europ J Mech A-Solid, 2009, 28: 1026-1034

4 Rebouillat S, Liksonov D. Fluid-structure interaction in partially filled liquid containers: A comparative review of numerical approaches. Comput Fluids, 2010, 39: 739-746

5 Zhu R Q, Fang Z Y, Zhang Z G, et al. Level-set method for pitching impact pressure induced by violent sloshing in a tank (in Chinese). J Ship Mech, 2008, 12: 344-351

6 Ibrahim R A. Liquid Sloshing Dynamics: Theory and Applications. New York: Cambridge University Press, 2005

7 Yue B Z. Large scale amplitude liquid sloshing in container under pitching excitation. Chinese Sci Bull, 2008, 53: 3816-3823

8 Yue B Z, Wang Z. Nonlinear phenomena of three-dimensional liquid sloshing in microgravity environ. Chinese Sci Bull, 2006, 51: 2425-2431

9 Gavrilyuk I, Lukovsky I, Trotsenko Y, et al. Sloshing in a vertical circular cylindrical tank with an annular baffle. Part 2. Nonliear resonant waves. J Eng Math, 2007, 57: 57-78

10 Cheng X D, Hu M Z, Wen J H. Dynamics characteristics of liquid sloshing in a transversely vibrating spherical tank with a spacer under low gravity. J Hydrodyn, 2008, 20: 762-769

11 He Y J, Ma X R, Wang B L. Stable response of low gravity liquid non-linear sloshing in a circle cylindrical tank. Appl Math Mech, 2007, 28: 1273-1285

12 Atwood B T, Schowalter W R. Measurements of slip at the wall during flow of high-density polyethylene through rectangular conduit. Rheol Acta, 1989, 28: 134-146

13 Horn R G, Vinogradova O I, Mackay M E, et al. Hydrodynamic slippage inferred from thin film drainage measurements in a solution of non-absorbing polymer. J Chem Phys, 2000, 112: 6424-6433

14 Migler K B, Hervet H, Léger L. Liquid sloshing dynamics. Phys Rev Lett, 1993, 70: 287-290

15 Vinogradova O I. Slippage of water over hydrophobic surfaces. Int J Miner Process, 1999, 56: 31-60

16 Lu K Q, Hou M Y, Wang Q, et al. The earthquake precursor detected in a granular medium and a proposed model for the propagation of precursive stress-strain signal. Chinese Sci Bull, 2011, 56: 1071-1079

17 Thompson P A, Troian S M. A general boundary condition for liquid flow at solid surfaces. Nature, 1997, 89: 360-362

18 Gupta S A, Cochran H D, Cummings P T. Shear behavior of squalane and tetracosane under extreme confinement. II Confined film structure. J Chem Phys, 1997, 107: 10327-10334

19 Yang W, Liu S H, Lin H. Viscous liquid sloshing damping in cylindrical container using a volume of fluid method. Sci China Ser E-Tech Sci, 2009, 52: 1484-1492

20 Wang L X, Zhou Q, Xu S Y. Investigation of the sliding friction behaviors of locust on slippery plates. Chinese Sci Bull, 2009, 54: 4549-4554

21 Vinogradova O I, Yakubov G E. Dynamic effects on force measurements. 2 Lubrication and the atomic force microscope. Langmuir, 2003, 19: 1227-1234

22 Zhou J F, Gu B Q, Shao C L. Boundary velocity slip of pressure driven liquid flow in a micron pipe. Chinese Sci Bull, 2011, 56: 1603-1610

23 Santra B, Dandapat B S, Anderson H I. Axisymmetric stagnation-point fow over a lubricated surface. Acta Mech, 2007, 194: 1-10

24 Li J, Zhou M, Cai L, et al. On the measurement of slip length for liquid flow over super-hydrophobic surface. Chinese Sci Bull, 2009, 54: 4560-4565

25 David W F, Standingford L, Schwartz W. A model for contact line energy dissipation for sloshing motions in partially filled containers. In: 37th AIAA Aerospace Sciences Meeting and Exhibit, Reno, NV, 1999

26 Bretherton F P. The motion of long bubbles in tubes. J Fluid Mech, 1960, 10: 166-188

Open Access This article is distributed under the terms of the Creative Commons Attribution License which permits any use, distribution, and reproduction in any medium, provided the original author(s) and source are credited. 